\title{
Use of St John's wort (Hypericum perforatum $L$ ) in members of a depression self-help organisation: a 12-week open prospective pilot study using the HADS scale.
}

\author{
Andrew G. Mayersa , David S. Baldwin ${ }^{a}$, Ruth Dyson ${ }^{a}$, \\ Richard W. Middleton ${ }^{b}$ and Amelia Mustaphac \\ aCommunity Clinical Sciences, School of Medicine, \\ University of Southampton, UK \\ bLichtwer Pharma UK Ltd, Marlow, UK \\ ${ }^{c}$ Depression Alliance, London, UK
}

Correspondence to Andrew Mayers, University Department of Psychiatry, University of Southampton, Royal South Hants Hospital, Brintons Terrace, Southampton SO14 0YG, UK

\section{Received}

Keywords: St John's wort, Hypericum, Depression, anxiety, HADS

\begin{abstract}
A B STRACT
The findings of randomised controlled trials confirm that St John's wort (Hypericum perforatum $L$ ) is effective in reducing symptoms in mild to moderate depression, but few studies have focused on the effect on anxiety symptoms. Most studies have employed observer-rated scales to measure treatment outcome, only few used self-rated scales. We sought to examine these issues in a cohort of members of the depression self-help organisation Depression Alliance UK ( $n=39)$. Hospital Anxiety and Depression (HADS) scales were used at baseline, prior to St John's wort administration, and for

subsequent assessments at weeks 4, 8 and 12 . A subject-rated version of the Clinical Global Impression - Improvement (CGI-I) scale was employed at follow-up assessments. HADS scores were significantly reduced after taking St John's wort, particularly for those reporting milder depression and more severe anxiety. HADS scores compared well with patient improvement ratings, indicating the feasibility of use in this population. Controlled studies are needed in a larger cohort to examine these findings, before definitive conclusions can be made.
\end{abstract}

better response rates than placebo $(52.7 \%$ vs $42.3 \%)$ and significantly greater reductions in HAMD scores (9.8 vs 7.1). Side effects and tolerability were similar between the groups. The mean HAMD (17-item) score at baseline in this study was 21.9. In an earlier study ${ }^{2}$, where baseline HAMD (21-item) scores averaged 19.45, there were greater differences between St John's wort and placebo. In that study, 159 depressed patients (ICD-10 diagnosis; HAMD 21-item score, 16-24) were randomised to St John's wort (ZE117) or placebo. Response rates were significantly better for St John's wort (56\%) than placebo (15\%), and reductions in HAMD scores were significantly greater in the active group (9.6 vs 0.8). Once more, side effects and tolerability were similar between the groups. The severity of depression at baseline appears to be an indicator for the efficacy of St John's wort. 
Two studies that failed to find a difference between St John's wort and placebo set higher thresholds for depression severity ${ }^{3,4}$, although it should be noted that in the Davison study $^{3}$ sertraline did not differ from placebo either. Both studies set an inclusion criterion of 20 on HAMD (17item). However, the consensus in trails involving St John's wort indicate that it is more efficacious in patients with mild to moderate depression. ${ }^{5}$

Other studies have compared St John's wort with traditional antidepressants. In one study ${ }^{6}, 30$ depressed patients (DSM-IV diagnosis; HAMD score $\geq 17$, version not specified) were randomised to St John's wort (LI 160) or sertraline for seven weeks. HAMD scores were significantly reduced in both groups, but did not differ from each other (St John's wort 8.4; sertraline 9.1), nor did they differ on response rates ( $47 \%$ vs $40 \%)$. Both groups reported similar side effects and tolerability. In a larger study ${ }^{7}, 240$ depressed patients (ICD-10 diagnosis; HAMD 21 score, 16-24) were randomised to St John's wort (ZE117; $n=126)$ or fluoxetine $(n=114)$ for six weeks. HAMD scores were slightly more reduced for St John's wort than fluoxetine (but not significantly), but overall response rates were significantly better for St John's wort $(P=0.005)$. Side effects were less common with St John's wort (8\%) than fluoxetine (23\%).

Comparative studies are not restricted to SSRIs; in one study 324 depressed patients (ICD-10 diagnosis; HAMD 17 score $\geq 18$ ) were randomised to $\mathrm{St}$ John's wort (ZE117; $n=157)$ or imipramine $(n=167)$ for six weeks ${ }^{8}$. HAMD reductions were similar between the groups (St John's wort, 10.4; imipramine, 9.4), although St John's wort was found to be superior in respect of anxiety-somatisation items. Side effects were less common with St John's wort (39\%) than imipramine (63\%); side effects led to withdrawal in $16 \%$ of those taking imipramine, and 3\% with St John's wort. Estimates of side effects with St John's wort range between 12\% and $38 \%$ of cases $^{9}$, with one meta-analysis suggesting around 20\%.5 Furthermore, St John's wort may interact adversely with traditional antidepressants. ${ }^{10,11}$

We previously conducted a retrospective analysis of data from members of the self-help organisation, Depression
Alliance (UK). ${ }^{12}$ One-half of the 452 subjects reported using St John's wort, $50 \%$ of whom reported improvement in depressive symptoms within four weeks. This was more pronounced for those with mild (compared to more severe) symptoms, and less noticeable for those who took St John's wort alongside other antidepressants. Side effects were reported by one-quarter of the St John's wort users.

We present prospective data that evaluate the effectiveness of St John's wort in a cohort of members of Depression Alliance, over a course of 12 weeks. There has been little focus on the effect of St John's wort on anxiety symptoms, although one study noted marked decreases in depressive agitation and anxiety symptoms with St John's wort. ${ }^{13}$ The relative benefits in respect of age and gender have also received little attention. Another aim of the study was to assess the feasibility of using the HADS in this population, with particular reference to the completion of scales, and the relation between HADS scores and scores on a subject-rated version of the CGI-I.

\section{Method}

\section{Subjects}

Members of the UK depression self-help organisation 'Depression Alliance' were contacted and supplied with the baseline HADS, to determine potential suitability for inclusion. Respondents taking medications known to interact with St John's wort, those with a history of self-harm or suicide, and pregnant or breastfeeding women were excluded. Thirty-nine subjects were included; the mean age was 43.69 (standard deviation, 13.07), range 22-78 years; there were 19 men and 20 women. Ethical approval was granted by the Southampton \& South West Hants Local Research Ethics Committee, in accordance with the principles of the Declaration of Helsinki (S39/00).

\section{Materials and procedure}

The subjects were sent supplies of St John's wort by the St John's wort Information Centre, Brackley, (LI 160, $300 \mathrm{mg}$ daily) and further HADS questionnaires to be completed after 4 , 8 and 12 weeks of the study. The sub- ject-rated version of the CGI-I scale was also completed at weeks 4, 8 and 12 .

Outcome measures in antidepressant studies are frequently examined using observer-rated scales. Patient-rated scales are used less frequently, but can provide valid and reliable data. The Hospital Anxiety and Depression Scale (HADS; ${ }^{14}$ ) was designed to elicit anxiety and depressive symptoms in medical patients. The scale is brief and simple to complete, employing 14 items ( 7 for each of anxiety and depressive symptoms). Furthermore, as the scale does not include severe symptoms, it may be better suited to milder forms of psychiatric illness $^{15}$ than more frequently used scales. In an extensive review by Herrmann ${ }^{15}$, it was established that HADS is well accepted by patients, with reported acceptance of around 95\%. Drawing on a wide range of studies, the HADS was shown to have good reliability, with internal consistencies ranging from 0.80 to 0.93 (Cronbach alphas) and high retest reliability $(r>0.80)$ for up to two weeks. Herrman's study also points to high validity, in respect of consistent factor analyses and high discriminant and concurrent validity.

The original authors ${ }^{14}$ recommended two cut-off points in respect of total HADS scores to determine levels of anxiety and depression: 8 for possible illness, 11 for probable illness. Herrmann ${ }^{15}$ reports several studies that reflect sensitivity and specificity of 0.8 or higher for these cut-off points to reliably detect those conditions. The author also reports that the HADS remains robust in both cross-sectional and longitudinal studies. In those studies, it was noted that women generally score higher than men on HADS anxiety, with no significant differences for gender on the depression subscale. HADS anxiety scores tended to be higher in those aged 30-59, and lower in those over 70 years. Meanwhile HADS depression scores tended to be highest in those aged 50-59, but lowest in those under 30 years.

The HADS has been used as an outcome measures in many randomised controlled trials. In the Hampshire Depression Project ${ }^{16}$ the effect of a comprehensive educational programme, targeted at primary care, was assessed using the HADS to examine clinical outcome. The HADS was also used to demonstrate the efficacy of escitalopram in treating anxiety and depression ${ }^{17}$, and 


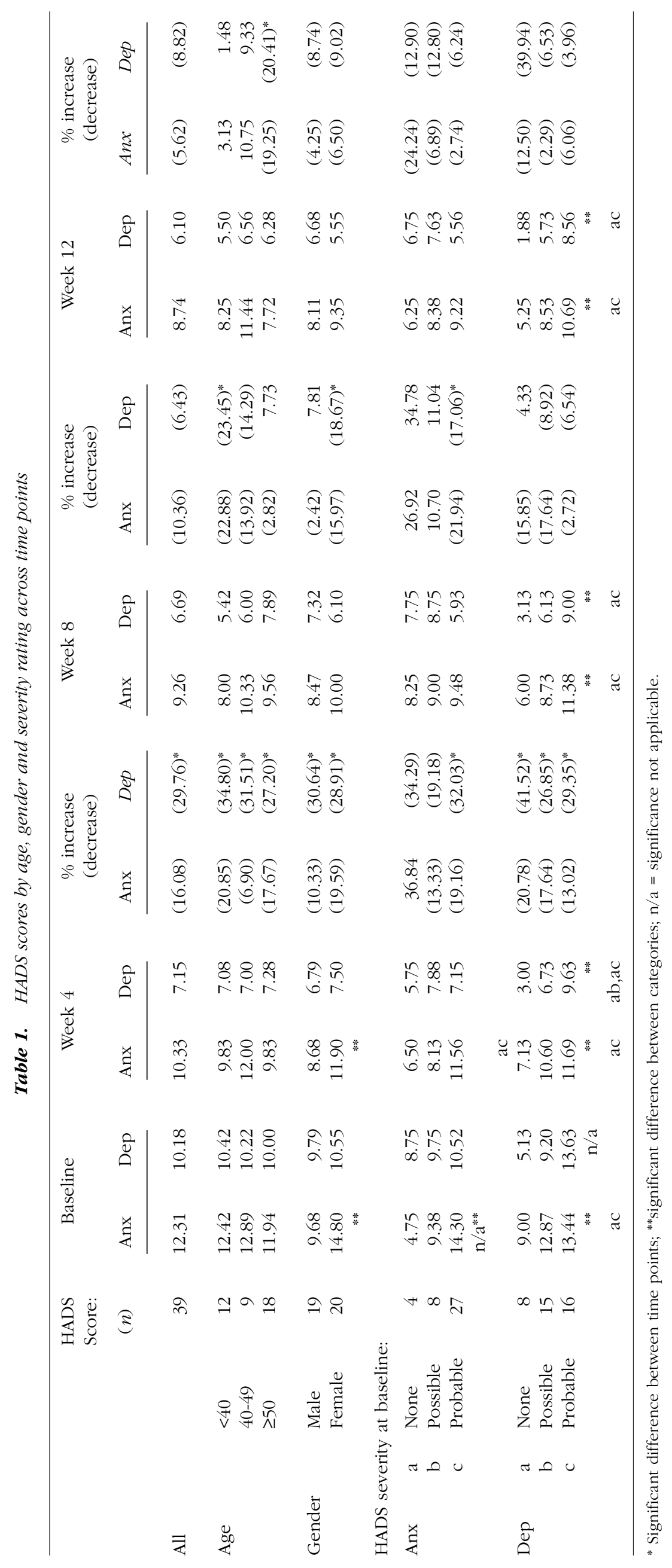


for examining the efficacy of venlafaxine in treating Generalised Anxiety Disorder $\left(\mathrm{GAD} ;{ }^{18}\right)$. There are few studies that examine the use of the scale in a depression self-help group, however one study ${ }^{19}$ investigated this in 341 members of the 'Fellowship of Depressives Anonymous', finding robust psychometric properties and good internal reliability in that population. No studies have evaluated the use of HADS during use of St John's wort. A subject-rated version of the Clinical Global Impressions - Improvement scale (CGI-I) ${ }^{20}$ was used to examine illness improvement at weeks 4, 8 and 12.

\section{Results}

Table 1 shows the HADS anxiety and depression scores for the sample. HADS anxiety scores were significantly reduced at both the four-week ( $t=$ 3.768 , df 38, $P=0.001)$ and eight-week assessments ( $t=2.266$, df 38, $P=0.029$ ). The HADS depression scores were significantly reduced at week $4(t=5.983$, df $38, P<0.001$ ).

A stepwise regression was conducted for HADS scores at each time point to establish which of the variables contributed most to the overall variance of those scores. In respect of baseline scores, HADS anxiety scores were predicted by gender and depression severity $\left(r^{2}=0.49 ; \mathrm{F}=17.179\right.$, df $2,38 P$ $<0.001$ ); no single variable uniquely contributed to HADS depression scores. At week 4, HADS anxiety scores were predicted by baseline severity ratings of anxiety and depression $\left(r^{2}=0.29 ; F=8.564\right.$, df 2, $38 P=0.001$ ), while only depression severity ratings at baseline predicted HADS depression scores at week $4\left(r^{2}=\right.$ 0.39; $\mathrm{F}=23.796$, df $1,38 P<0.001)$. At week 8 , HADS anxiety scores were predicted by baseline ratings of depression severity $\left(r^{2}=0.23 ; \mathrm{F}=11.164\right.$, df $1,38 P$ $=0.002$ ), while baseline severity ratings of anxiety and depression, and age, predicted HADS depression scores at week $8\left(r^{2}=0.43 ; F=10.617\right.$, df $3,38 P<$ 0.001). At 12-week follow-up, HADS anxiety scores were predicted by baseline ratings of depression severity $\left(r^{2}=\right.$ 0.19; $F=8.830$, df $1,38 P=0.005$ ), while baseline severity ratings of anxiety and depression predicted HADS depression scores at week $12\left(r^{2}=0.39 ; F=13.153\right.$, df 2, $38 P<0.001)$.
Main effects for between-group analyses were conducted using one-way ANOVA. There was no effect for age group for either of the HADS scores at any time, but women reported significantly higher HADS anxiety scores than men at baseline $(F=28.857$, df $1,38 P<$ $0.001)$ and week $4(F=7.488$, df $1,38 P$ $=0.009$ ). There was a significant effect for baseline severity of anxiety on HADS anxiety scores at week $4(F=5.399$, df 2 , $38 P=0.009)$, with those rated as probably anxious at baseline returning significantly higher HADS anxiety scores at week 4, than those rated as having no clinical evidence of anxiety (normal) at baseline ( $P=0.042)$. However, there was a significant effect for baseline ratings of depression severity on HADS anxiety and depression scores at all follow-up assessments, and for HADS anxiety scores at baseline. Those rated as probably depressed reported significantly higher HADS depression scores, than those rated as normal, throughout the study.
The change in HADS scores between time points, for each sub-category of variable, was assessed using pairedsamples $t$-tests. In subjects aged under 40 years, HADS anxiety and depression scores were significantly reduced at week $4(t=2.400$, df $11, P=0.035$; and $t=3.895$, df $11, P=0.002$ respectively) and week $8(t=2.276$, df $11, P=0.044$; $t=2.345$, df $11, P=0.039)$. These were also significantly reduced at week 4 $(t=3.059$, df 17, $P=0.007 ; t=5.030, \mathrm{df}$ $17, P<0.001)$ and week $12(t=2.547$, df $17, P=0.021 ; t=3.757$, df $17, P=$ 0.002 ) for those aged 50 years and over. However, both HADS scores remained relatively stable for those aged 40 to 49 years. For female subjects, HADS anxiety and depression scores were significantly reduced at week $4(t=$ 3.591, df 19, $P=0.002 ; t=3.814$, df 19 , $P=0.001)$ and week $8(t=3.535$, df 19 , $P=0.002 ; t=2.833$, df $19, P=0.011)$, while the only notable change for men was a significant reduction in HADS

Table 2. HADS scores by CGI-Improvement rating

\begin{tabular}{|c|c|c|c|c|c|c|c|c|}
\hline & \multicolumn{2}{|c|}{ HADS score: } & \multicolumn{2}{|c|}{ Baseline } & \multicolumn{2}{|c|}{ Week 4} & \multicolumn{2}{|c|}{$\begin{array}{l}\% \text { increase } \\
\text { (decrease) }\end{array}$} \\
\hline & & $(n)$ & Anx & Dep & Anx & Dep & Anx & Dep \\
\hline \multirow[t]{9}{*}{ CGI-I F1 } & 1 & 0 & & & & & & \\
\hline & 2 & 9 & 11.22 & 7.89 & 7.22 & 4.00 & $(35.65)^{*}$ & $(49.30)^{*}$ \\
\hline & 3 & 13 & 12.69 & 9.62 & 10.38 & 6.54 & $(18.20)^{*}$ & $(32.02)^{*}$ \\
\hline & 4 & 14 & 13.29 & 11.86 & 12.29 & 9.64 & $(7.52)$ & $(18.72)^{*}$ \\
\hline & 5 & 3 & 9.33 & 11.67 & 10.33 & 7.67 & 10.72 & (34.28) \\
\hline & 6 & 0 & & & & & & \\
\hline & 7 & 0 & & & & & & \\
\hline & & & \multicolumn{2}{|c|}{ Week 4} & \multicolumn{2}{|c|}{ Week 8} & \multicolumn{2}{|c|}{$\begin{array}{l}\% \text { increase } \\
\text { (decrease) }\end{array}$} \\
\hline & & & Anx & Dep & Anx & Dep & Anx & Dep \\
\hline \multirow[t]{9}{*}{ CGI-I F2 } & 1 & 3 & 9.33 & 5.33 & 5.67 & 2.33 & $(39.23)$ & $(56.29)^{*}$ \\
\hline & 2 & 8 & 6.75 & 4.63 & 5.38 & 3.88 & $(20.30)^{*}$ & (16.20) \\
\hline & 3 & 13 & 11.15 & 7.46 & 9.46 & 6.46 & $(15.16)^{*}$ & (13.40) \\
\hline & 4 & 12 & 11.42 & 8.08 & 11.08 & 8.58 & (2.98) & 6.19 \\
\hline & 5 & 3 & 13.00 & 10.67 & 15.00 & 12.00 & 15.38 & 12.46 \\
\hline & 6 & 0 & & & & & & \\
\hline & 7 & 0 & & & & & & \\
\hline & & & \multicolumn{2}{|c|}{ Week 8} & \multicolumn{2}{|c|}{ Week 12} & \multicolumn{2}{|c|}{$\begin{array}{l}\text { \% increase } \\
\text { (decrease) }\end{array}$} \\
\hline & & & Anx & Dep & Anx & Dep & Anx & Dep \\
\hline \multirow[t]{7}{*}{ CGI-I F3 } & 1 & 7 & 6.14 & 2.29 & 3.57 & 1.29 & $(41.86)$ & $(43.67)^{*}$ \\
\hline & 2 & 8 & 7.88 & 5.50 & 6.75 & 3.88 & (14.34) & $(29.45)$ \\
\hline & 3 & 11 & 9.82 & 7.27 & 10.73 & 7.00 & 9.27 & $(3.71)$ \\
\hline & 4 & 9 & 10.00 & 8.67 & 9.22 & 8.11 & $(7.80)$ & $(6.46)$ \\
\hline & 5 & 4 & 14.25 & 10.75 & 15.25 & 12.00 & 7.02 & 11.63 \\
\hline & 6 & 0 & & & & & & \\
\hline & 7 & 0 & & & & & & \\
\hline
\end{tabular}

CGI-I scores: 1, Very much improved; 2, Much improved; 3, Slightly improved;

4, No change in symptoms; 5, Slightly worse; 6: Much worse; 7, Very much worse.

* Significant difference between time points. 
depression scores at week $4(\underline{t}=4.757$, df 18, $P<0.001$ ).

Severity ratings of anxiety and depression demonstrated quite different profiles to one another. For those rated as probably anxious (severe) at baseline, HADS anxiety and depression scores were significantly reduced at week $4(t=4.564$, df 26, $P<0.001 ; t=$ 5.432, df 26, $P<0.001)$ and week $8(t$ $=4.786$, df 26, $P<0.001 ; t=2.855$, df $26, P=0.008)$. All other ratings of anxiety severity showed no differences between time points. For subjects rated as normal and as 'probably depressed' (severe) on depression severity, HADS depression scores remained relatively stable, except for a significant decrease in depression scores at week 4 (normal: $t=2.374$, df 7, $P=0.049$; probable $t=4.404$, df $15, P=0.001$ ). For those rated as possibly depressed (mild to moderate), decreases in HADS score were more widespread, with significant decreases in HADS anxiety and depression scores at week $4(t=2.622$, df $14, P=0.020 ; t=2.412$, df $14, P=$ $0.004)$ and in anxiety scores at week 8 $(t=2.272$, df $14, P=0.039)$.

Changes in HADS scores were compared to scores on the subject-rated version of the CGI-I ratings (Table 2). At week 4 no subjects were 'very much improved', but HADS anxiety and depression scores were significantly reduced for those who were 'much improved' ( $t=4.000$, df $8, P=0.004$; $t=3.865$, df $8, P=0.005)$ and 'slightly improved' ( $t=4.629$, df $12, P=0.001$; $t=3.682$, df $12, P=0.003)$. Depression scores were also significantly reduced for those who reported 'no change in symptoms' ( $t=2.987$, df $13, P=0.012$ ). At week 8 , those who were 'very much improved' showed decreases in HADS anxiety and depression scores, which was significant for the depression scores ( $t=5.196$, df $2, P=0.035)$. There was a significant reduction in HADS anxiety scores for those 'much improved' ( $t=$ 2.434, df 7, $P=0.045$ ) and 'slightly improved' ( $t=2.587$, df $7, P=0.024)$. For those subjects who showed no improvement, or who were 'slightly worse', HADS anxiety and depression scores generally increased (although not significantly). At week 12, both HADS scores were much improved for those 'very much improved', fairly reduced for those 'much improved', somewhat reduced for those 'slightly improved' or who showed 'no improvement', while
HADS anxiety and depression scores increased for those subjects rated as being 'slightly worse' on CGI-I; although none of these changes were significant.

\section{Discussion}

Following the administration of St John's wort, self-rated anxiety was significantly reduced at weeks 4 and 8, and depression at week 4. HADS anxiety and depression scores were significantly reduced at weeks 4 and 8 for those aged under 40, and at weeks 4 and 12 for those aged 50 or over; there was no effect for those aged 40 to 49 . HADS anxiety and depression scores were significantly reduced for women at weeks 4 and 8 , and women reported significantly higher HADS scores than men at baseline and week 4 . There were no consistent effects for men. HADS anxiety and depression scores were significantly reduced at weeks 4 and 8 for those reporting the most severe anxiety at baseline, while no effect was found for those with mild, or no, anxiety at baseline. HADS sub-scale scores were significantly reduced at week 4 , and HADS anxiety at week 8 , for those reporting milder depression at baseline, while no consistent effect was found in this respect for those with more severe, or no, depression at baseline. Furthermore, while there was a significant difference in HADS anxiety scores, between those reporting most anxiety and those reporting no anxiety at week 4 , this effect was no longer apparent at weeks 8 and 12 . However, a significant difference in both HADS scores, between those reporting most depression and those reporting none, remained throughout the study. Changes in HADS scores were generally reflected by change in scores on the global ratings.

These results suggest that St John's wort can be successful in reducing selfreported symptoms of depression and anxiety, particularly for those reporting more severe symptoms of anxiety and milder symptoms of depression. This supports previous evidence that St John's wort is more effective in mild to moderate depression', rather than more severe forms. ${ }^{3,4}$ The stepwise regression analysis indicated that depression severity at baseline was a constant predictor of HADS score at follow-up visits, supporting evidence that the efficacy of St John's wort can depend on baseline depression severity ${ }^{1-4}$. The suggestion that St John's wort might provide greater benefit for those most anxious has received little attention before, although one study reported that St John's wort reduced depressive agitation and anxiety ${ }^{13}$. Women scored more highly on HADS anxiety scores than men at baseline and week 4, which supports data from previous findings ${ }^{15}$. Those scores were significantly reduced at weeks 4 and 8, lending support to the finding that St John's wort benefits those with more severe anxiety.

Caution is warranted when interpreting findings from a small sample. There were a number of findings of sufficient magnitude to merit examination in a larger cohort. It is possible that different extracts of St John's wort result in different outcomes, and the precise extracts needs to be specified. Concurrent medication taken by the participants was not recorded; not only is there evidence of adverse interactions between St John's wort and traditional antidepressants ${ }^{10,11}$, and poorer outcomes $^{12}$, there is no certainty that outcomes here were not the result of a medication other than St John's wort. Furthermore, reported adverse events were not recorded and these need to be documented adequately.

Nevertheless, this pilot study produced some useful findings that could be investigated more comprehensively in a larger study. The findings here confirmed the body of evidence that St John's wort is efficacious to individuals reporting mild to moderate depression. It adds a tentative finding that this may also be possible in individuals with more severe anxiety. The HADS scale was successfully used in this depression self-help group population.

\section{Acknowledgement}

This study was supported by a research grant from Lichtwer Pharma.

\section{References}

1. Lecrubier Y, Clerc G, Didi R, Kieser M. Efficacy of St. John's Wort Extract WS 5570 in Major Depression: A DoubleBlind, Placebo-Controlled Trial. Am J Psychiatry 2002; 159:1361-1366. 
2. Schrader E, Meier B, Brattström A. Hypericum Treatment of Mild-Moderate Depression in a Placebo-Controlled Study. A Prospective, Double-Blind, Randomized, Placebo-Controlled, Multicentre Study. Hum Psychopharmacol Clin Exp 1998; 13:163-169.

3. Davidson RT, Hypericum Depression Trial Study Group. Effect of Hypericum perforatum (St John's Wort) in Major depressive Disorder: A Randomized Controlled Trial. JAMA 2002; 287:1807-1814.

4. Shelton R, Keller MB, Gelenberg A Dunner DL, Hirschfeld R, Thase ME et al. Effectiveness of St John's Wort in Major Depression: A Randomised Controlled Trial. JAMA 2001; 285:1978-1986.

5. Linde K, Ramirez G, Mulrow CD, Pauls A, Weidenhammer W, Melchart D. St. John's wort for depression - an overview and meta analysis of randomised clinical trials. BMJ 1996; 313:253-258.

6. Brenner R, Azbel V, Madhusoodanan S, Pawlowska M. Comparison of an Extract of Hypericum (LI160) and Sertraline in the Treatment of Depression: A DoubleBlind, Randomized Pilot Study. Clin Ther 2000; 22:411-419.

7. Schrader E. Equivalence of St John's wort extract (ZE117) and fluoxetine: A randomized, controlled study in mild-moder- ate depression. Int Clin Psychopharmacol 2000; 15:61-68.

8. Woelk H. Comparison of St John's wort and imipramine for treating depression; randomised controlled trial. BMJ 1999; 321:536-539.

9. Ernst E, Rand JI, Barnes J, Stevinson C. Adverse effects of the herbal antidepressant St. John's wort (Hypericum perforatum L.). Eur J Clin Pharmacol 1998; 54:589-594.

10. Gordon JB. Selective serotonin reuptake inhibitors and St. John's wort: possible toxicity? Am Fam Physician 1998; 57:950.

11. Wheatley D. St. John's wort: more knowledge needed. Pharm J 2000; 265:49.

12. Dyson R, Baldwin DS, Mayers AG, Tiernan A, Jenkins G. The reported use and effectiveness of Hypericum (St John's wort) on affective symptoms in a depression self-help group. Primary Care Psychiatry 2002; 8:99-102.

13. Friede M, Henneicke von Zepelin $\mathrm{HH}$, Freudenstein J. Differential therapy of mild to moderate depressive episodes (ICD-10 F 32.0; F 32.1) with St John's wort. Pharmacopsychiatry 2001; 34 (Suppl 1):S38-S41.

14. Zigmond AS, Snaith RP. The hospital anxiety and depression scale. Acta Psychiatr Scan 1983; 67:361-370.
15. Herrmann C. International experiences with the Hospital Anxiety and Depression Scale - a review of validation data and clinical results. J Psychosom Res 1997; 42:17-41.

16. Thompson C, Kinmouth AL, Stevens L, Peveler RC, Stevens A, Ostler KJ et al. Effects of a clinical-practice guideline and practice-based education on detection and outcome in primary care: Hampshire Depression Project randomised controlled trial. Lancet 2000; 355:185-191.

17. Baldwin DS. Escitalopram - in the treatment of mood and anxiety disorders. Eur Psychiatry 2002; 17 (Suppl 1):40.

18. Katz IR, Reynolds III CF, Alexopoulus GS, Hackett D. Venlafaxine ER as a treatment for generalized anxiety disorder in older adults: pooled analysis of five randomised placebo-controlled clinical trials. J Am Geriatr Soc 2002; 50:18-25.

19. Dagnan D, Chadwick P, Trower P. Psychometric properties of the Hospital Anxiety and Depression Scale with a population of members of a depression selfhelp group. Br J Med Psychol 2000; 73:129-137.

20. Guy W. ECDEU Assessment Manual of Psychopharmacology. Washington, DC: Department of Health, Education and Welfare, 1976. 